\title{
Simulated production rates of exotic nuclei from the ion guide for neutron-induced fission at IGISOL
}

\author{
Kaj Jansson ${ }^{\mathrm{a}}$, Ali Al-Adili, Nicklas Nilsson, Martin Norlin, and Andreas Solders ${ }^{\mathrm{b}}$ \\ Department of Physics and Astronomy, Uppsala University, Box 516, 75120 Uppsala, Sweden
}

Received: 11 September 2017 / Revised: 21 November 2017

Published online: 22 December 2017

(c) The Author(s) 2017. This article is published with open access at Springerlink.com

Communicated by A. Jokinen

\begin{abstract}
An investigation of the stopping efficiency of fission products, in the new ion guide designed for ion production through neutron-induced fission at IGISOL in Jyväskylä, Finland, has been conducted. Our simulations take into account the new neutron converter, enabling measurements of neutron-induced fission yields, and thereby provide estimates of the obtained yields as a function of primary proton beam current. Different geometries, targets, and pressures, as well as models for the effective charge of the stopped ions were tested, and optimisations to the setup for higher yields are suggested. The predicted number of ions stopped in the gas lets us estimate the survival probability of the ions reaching the downstream measurements stations.
\end{abstract}

\section{Introduction}

Nuclear-physics studies at the IGISOL-JYFLTRAP facility have a long-standing tradition where plenty of exotic nuclei have been explored [1]. In order to facilitate these investigations, neutron-rich isotopes have been produced via nuclear fission. A beam of protons, with energies above the Coulomb barrier, impinges on a fissionable target to produce highly charged fission products (FPs). Some of these will escape the target and eventually thermalise in a helium buffer gas, in the so-called fission ion guide. In this process, the initially highly charged FPs recombine and, due to the high ionisation potential of the helium atoms, a large fraction end up as singly charged ions. These can then be extracted and transported to the different experiments.

A requirement for the study of independent fission yields at IGISOL is that the stopping efficiency of the ion guide is free from, or can be corrected for, any systematic effects related to the ion species and their kinetic energies. A previous simulation driven investigation of the protoninduced fission ion guide [2], studied the stopping of FPs as a function of multiple experimental parameters (e.g., gas pressure and target thickness). The main finding was a small mass-dependent variation in the efficiency, within the systematic uncertainties of the simulations.

\footnotetext{
a e-mail: kaj.jansson@physics.uu.se

b e-mail: andreas.solders@physics.uu.se
}

As part of a recent upgrade of IGISOL, a $\mathrm{Be}(\mathrm{p}, \mathrm{xn})$ neutron-converter has been developed together with a dedicated ion guide for the study of neutron-induced fission [3]. The purpose of this is both to facilitate measurements of neutron-induced fission-yields [4] and to provide exotic nuclei for spectroscopy. As an example, studying neutron-rich isotopes in the tin region (masses, half lives, excited states, and so on) is important to understand the $r$-process [5], a mechanism to create nuclei heavier than iron through cosmological nucleosynthesis. Previous experiments, using neutron-induced fission, reported a decrease of the atomic charge, averaged over an isobar, of 0.88 , i.e., a relative higher yield of neutron-rich nuclei, compared to the proton-induced case [6]. In fig. 1 the predicted yields of tin isotopes from the ${ }^{238} \mathrm{U}(\mathrm{n}, \mathrm{f})$ and ${ }^{238} \mathrm{U}(\mathrm{p}, \mathrm{f})$ reactions are compared, using typical energies for the incoming particles at IGISOL. In fig. 2 the average $N / Z$ ratio of FPs is plotted as a function of the total neutron emission. This shows that going from protoninduced to neutron-induced fission increases the production of neutron-rich nuclei.

To study the behaviour of the neutron-induced ion guide, a new simulation tool-kit has been developed. Three different simulation packages were used: MCNPX [7] was used to calculate the neutron fluxes and spectra in the ion guide; the FPs was sampled from the fission observables distributions calculated by GEF [8] for each incident neutron energy; and Geant4 [9] was used to create a simulation software for the transport of FPs in the ion guide. 


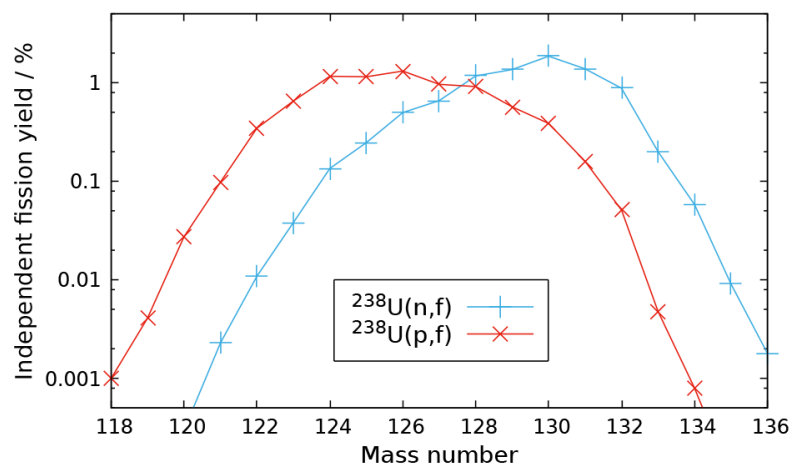

Fig. 1. The independent fission yields of tin isotopes from neutron- and proton-induced fission of ${ }^{238} \mathrm{U}$, respectively. The yields were calculated by the GEF code for incoming $12 \mathrm{MeV}$ neutrons and $25 \mathrm{MeV}$ protons, respectively.

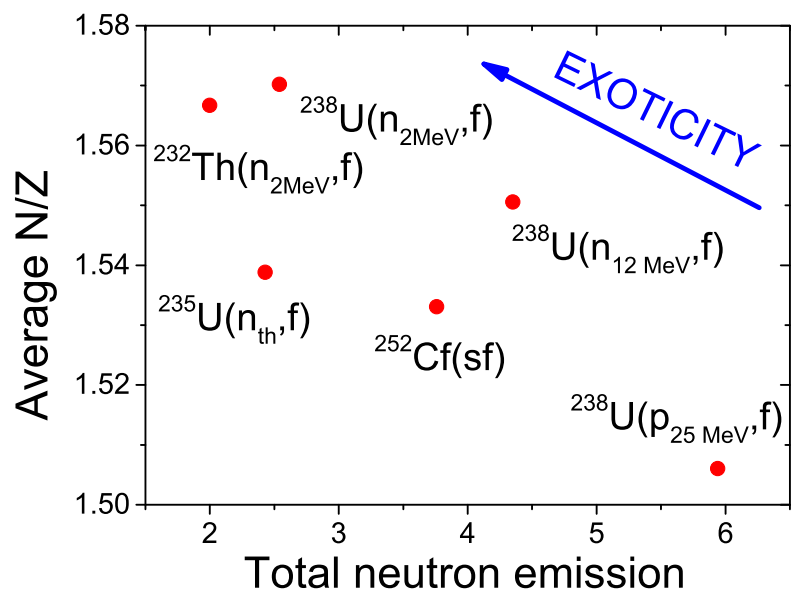

Fig. 2. The average $N / Z$ ratio as a function of the total neutron emission calculated by GEF. The average energy of the incoming fission neutron at IGISOL is $12 \mathrm{MeV}$.

\section{Experimental setup}

In this work, we have used an existing experimental setup as our starting point, and then varied some of the parameters. This experimental setup has been used in a measuring campaign in December 2016. The experiment will only be briefly described here, but more details are available in ref. [10].

The primary beam of $30 \mathrm{MeV}$ protons, impinge on a thick water-cooled beryllium target which converts the mono-energetic protons into a neutron spectrum covering the energy range from thermal to $28.4 \mathrm{MeV}$. To benefit from maximum neutron flux the ion guide is placed close to the water-cooled target.

The main part of the ion guide is a cylinder, $70 \mathrm{~mm}$ long and $60 \mathrm{~mm}$ in diameter, which is gas-filled and holds the uranium targets. The ion guide is continuously flushed with fresh helium gas at a nominal pressure of 200 mbar. However, during the measurement in December 2016 a higher pressure of 400 mbar was used. Thus, in order to make comparisons to the experimental results, we also conducted a simulation at this elevated pressure. A dif-

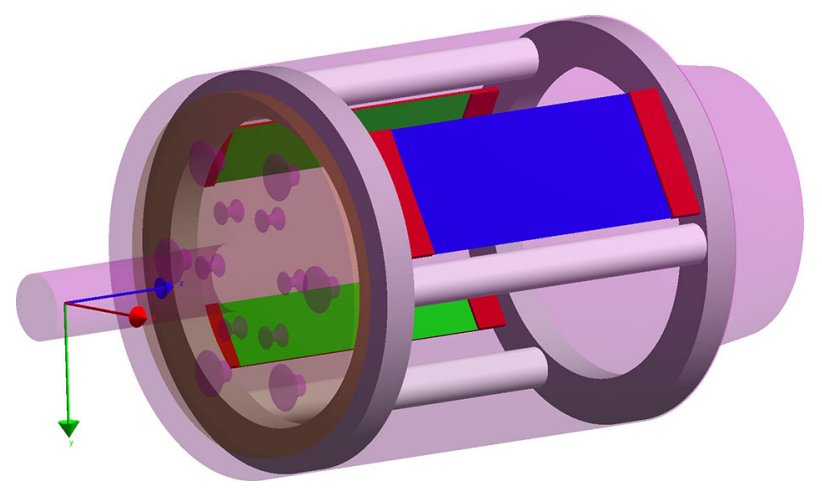

Fig. 3. 3D-rendering of the simulated geometry. The outer walls have been rendered transparent, and the gas filled inside was rendered translucent purple. Also the orange gas diffuser has been rendered translucent to improve visibility. The beam direction is perpendicular to the ion guide axis, along the $x$-axis (red arrow). The gas flow purging the ion guide is along the ion guide axis that coincides with the $z$-axis (blue arrow). The setup shown is the same that was used in the measurements in December 2016 with two uranium targets (green) and one titanium foil in the back (blue).

fuser at the inlet distributes the gas in the ion guide. The produced FPs will escape the target, be stopped in the gas, and follow the gas-flow out of the ion guide, where they will be accelerated and further transported. Two targets are held by a hexagonal target holder, close to the neutron source.

Both targets are $50 \mathrm{~mm} \times 10 \mathrm{~mm}$ rolled foils of natural uranium with a thickness of $15 \mathrm{mg} / \mathrm{cm}^{2}$. The holder has room for up to six targets, but only two were used during the December campaign. However, the experimental run also featured a titanium implantation foil, kept in place in the back in one of the target slots.

\section{Methodology}

Our reference simulation mimics the setup of the December experimental run, but uses the lower nominal helium pressure of 200 mbar. A 3D-rendering of the simulated geometry is depicted in fig. 3. Two other geometries were also simulated in order to investigate the potential benefits of including more small targets or using one big target. Cross sectional sketches of the three setups are depicted in fig. 4.

The main simulation software is based on the Geant4 library [9], but the neutron flux generated by the primary beam was calculated using MCNPX [7] and mass and energy distributions of the FPs were provided by GEF [8].

\subsection{The neutron source}

The neutron source has been extensively described and characterised elsewhere [3] and hence, only a brief description will be given here. The source consists of a $6 \mathrm{~mm}$ thick and $50 \mathrm{~mm}$ in diameter beryllium disc cooled by water on 

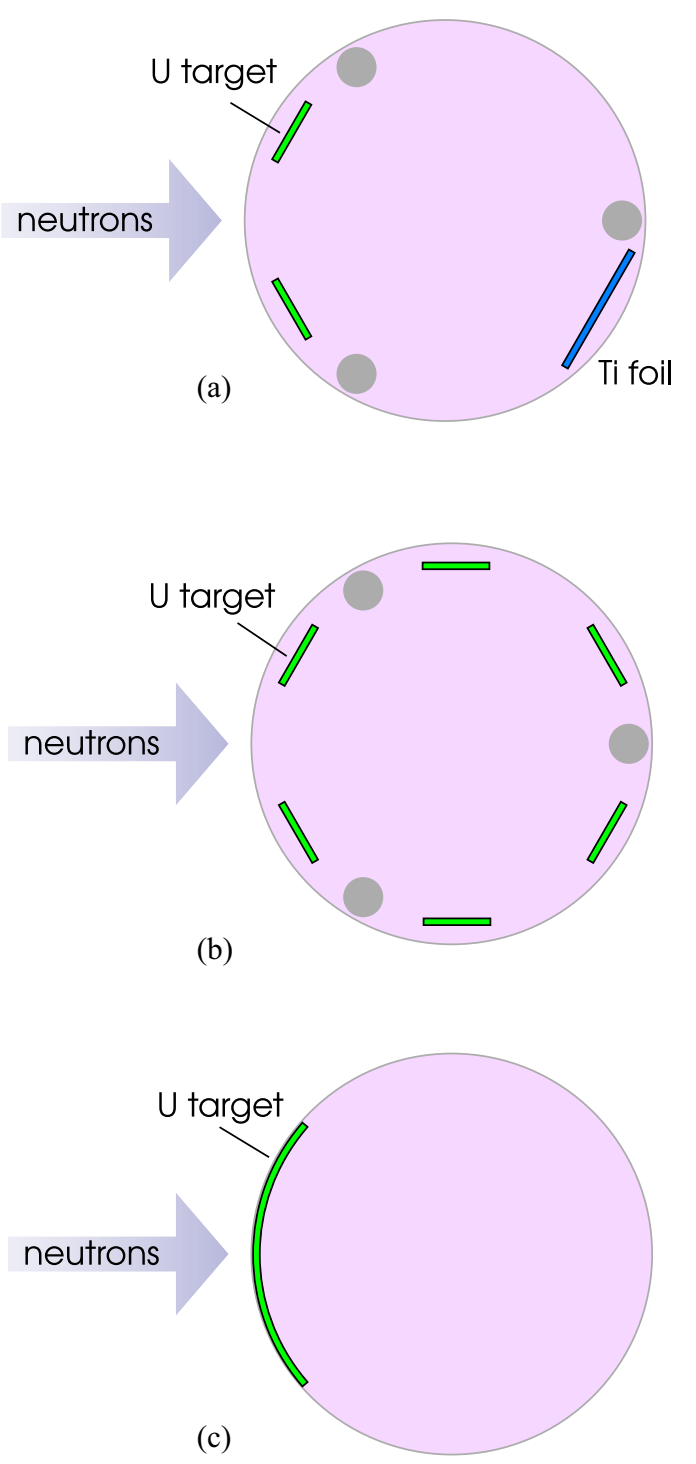

Fig. 4. Cross sectional schematics of the target placement for the different setups. The gas flow goes out of the plane of the drawings. (a) Reference geometry used in the measurements in December 2016. (b) Geometry with six uranium targets. (c) Geometry with one big uranium foil pressed against the inside of the ion guide.

the back side. High energy protons are delivered from either of two cyclotrons at currents ranging up to $200 \mu \mathrm{A}$. Through the $\mathrm{Be}(\mathrm{p}, \mathrm{xn})$ reactions, a neutron field with energies ranging from thermal almost up to the full proton energy, is created.

The neutron source was simulated using the Monte Carlo code MCNPX version 2.5.0 [7]. The simulation starts with a beam of protons at $30 \mathrm{MeV}$. The proton interaction with the beryllium disk is modelled using the ENDF70PROT library, based on ENDF/B-VII.0 evaluation [11]. The neutron transport through the beryllium is based on the la150n library from the LANL evaluation [12], that extends up to $100 \mathrm{MeV}$. The evolution of the neutron field through the rest of the geometry, consisting of the holder for the beryllium, the cooling water,

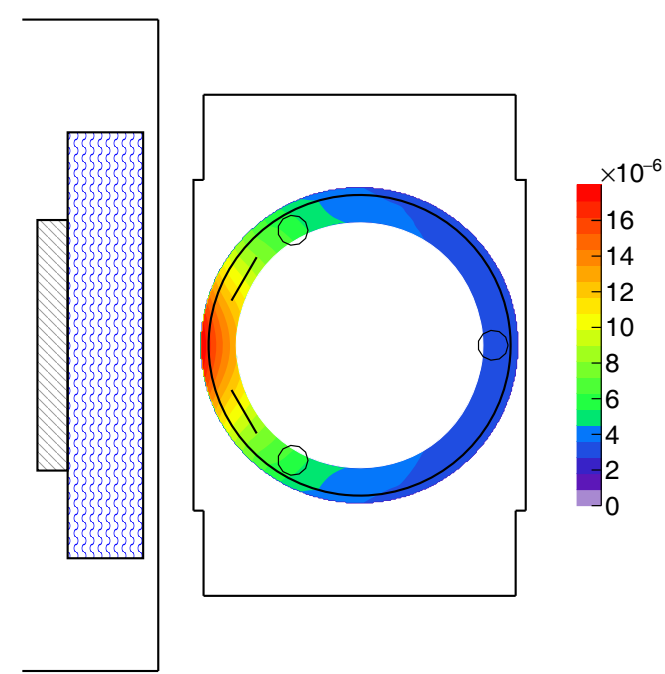

Fig. 5. The neutron flux in $\mathrm{n} /\left(\mathrm{cm}^{2} \mu \mathrm{Cs}\right)$, on top of a schematic of the ion guide. The flux was only recorded in a cylindrical shell enclosing the target positions. To the left of the ion guide is the neutron source. The beryllium target (grey striped pattern) is cooled by the water (blue wave pattern).

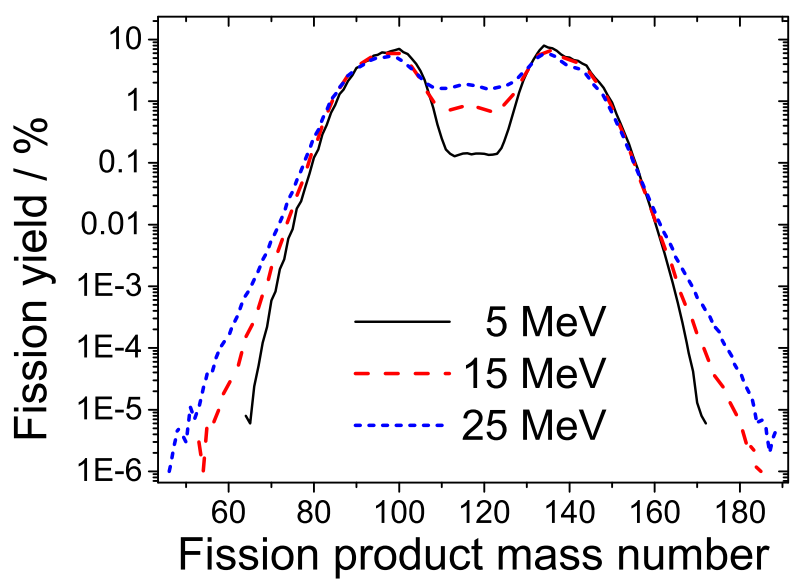

Fig. 6. Mass yields of ${ }^{238} \mathrm{U}(\mathrm{n}, \mathrm{f})$ from GEF calculations at 5, 15 and $25 \mathrm{MeV}$ incident neutron energy. Each yield is normalised to $200 \%$.

the gas filled ion guide and the vacuum chamber, makes use of a combination of the endf70a [11], the endf66a [13] or the endl92 [14] libraries, depending on the material.

The neutron flux was recorded in a $7 \mathrm{~mm}$ thick and $54 \mathrm{~mm}$ long cylindrical shell, enclosing the lateral surface of the ion guide and all tested target positions. A total of 30 cylindrical mesh tallies were used, one for each integer energy from 0 to $30 \mathrm{MeV}$. In fig. 5 the obtained neutron flux, integrated over all energies is depicted on top of a schematic of the ion guide.

\subsection{Fission product generation and transport}

Version 2016/1.2 of the GEF [8] code was used to obtain realistic ion distributions. Due to the yield dependence on neutron energy (fig. 6), the GEF calculations were performed at 31 different energies, ranging from 0 to $30 \mathrm{MeV}$ (in steps of $1 \mathrm{MeV}$ ). 
For each event, a FP was sampled from the isotopic distributions obtained with GEF. Once the ion species was settled, the initial energy was also sampled based on the GEF output. The position was sampled uniformly in the available target volume, even though fission is more likely to happen where the neutron flux is greater. This was corrected post-simulation as described later. The initial momentum directions were isotropically sampled, assuming negligible neutron momentum transfer and anisotropy.

The heavy ion was transported by the simulation code until all its energy was lost. To ensure sufficient granularity we limited the particle step length to $1 \mathrm{~mm}$ and $50 \mathrm{~nm}$ in the gas and the solid materials, respectively. Its initial state as well as final position were recorded into a ROOTfile [15]. Note that only one, not two, FP was simulated for each event. Every simulation sampled $1 \times 10^{8}$ events in total.

In addition to the different geometries, a few variations of the simulation parameters were tested. The nominal gas pressure for most experiments at IGISOL is $200 \mathrm{mbar}$ but an increase in the gas pressure $(400 \mathrm{mbar}$, as used in the experiment in December 2016) was also simulated, as well as $600 \mathrm{mbar}$ and $800 \mathrm{mbar}$ to investigate the pressure dependence. The use of both thinner $\left(7.5 \mathrm{mg} / \mathrm{cm}^{2}\right)$ as well as much thicker $\left(150 \mathrm{mg} / \mathrm{cm}^{2}\right)$ uranium targets was investigated. To test part of the model dependence of the stopping calculation, the effective charge of the ions was calculated using two different models.

The influence of the effective charge model used was tested by comparing the model of Schiwietz et al. [16] to the standard Geant4 model, which is based on the work of Ziegler et al. [17,18]. The model of Schiwietz et al. has previously been found [19] to agree better with experimental data from LOHENGRIN than the standard Geant 4 model. The source code of the Geant 4 class G4ionEffectiveCharge was edited, in order to allow for a non-standard model.

\subsection{Analysis}

The initial position of the ions were uniformly sampled from the target volume, but the neutron flux is a function of the spatial coordinates and thus, so is the fission rate. In fig. 7 the simulated average flux, cross section and fission rate are depicted. The average neutron energy for the fission events were $11.8 \mathrm{MeV}$. The highest flux is found close to the neutron source and close to the beam axis. To remedy the unrealistic sampling each event was given a weight based on the fission rate.

The macroscopical cross section, $\Sigma=\sigma N_{\mathrm{U}}$, is the product of the microscopical cross section $\sigma$ (taken from the ENDF/B-VII.1 evaluation [11]), and the total number of uranium atoms in the target $N_{\mathrm{U}}$. The weighting factor $w$ was constructed for each event, by multiplying the neutron-energy dependent $\Sigma$, with the position dependent flux $\phi$, and dividing with the number of simulated events $N$,

$$
w=2 \frac{\Sigma \phi}{N}
$$
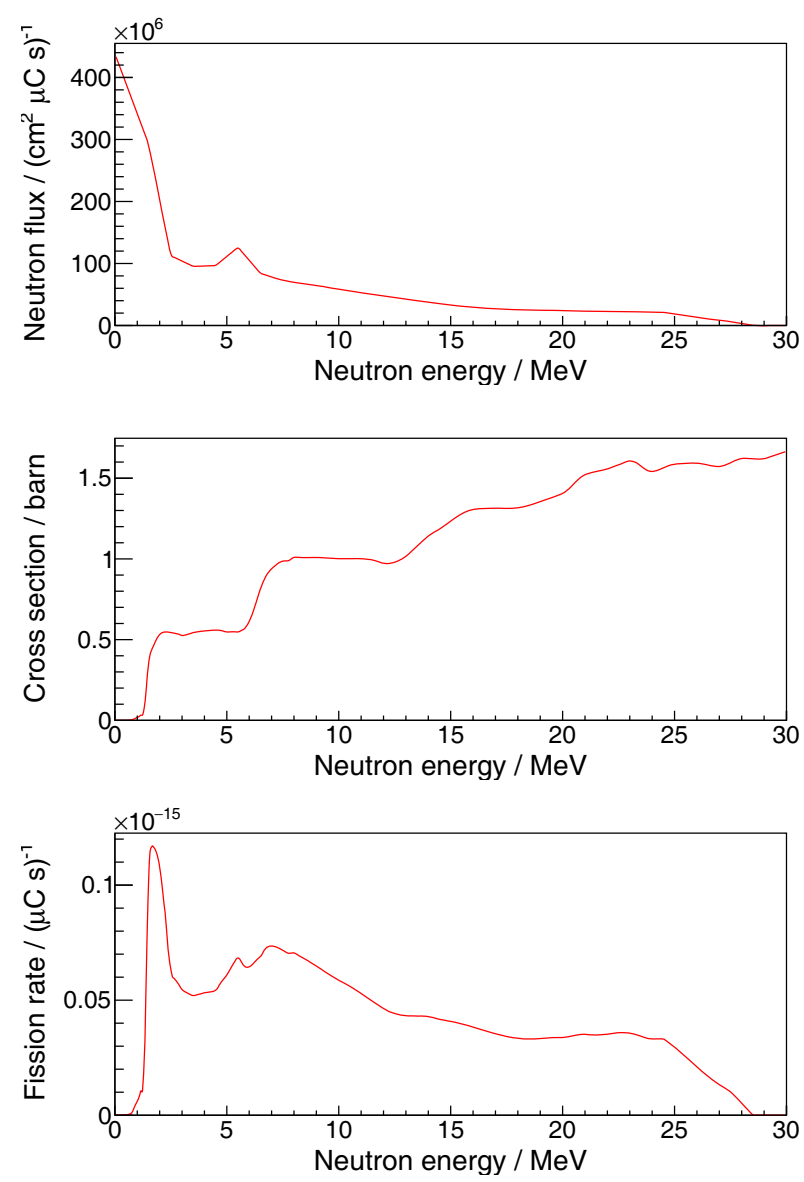

Fig. 7. The average neutron flux, the fission cross section, and the average fission rate (the product of the two previous) as functions of the neutron energy.

where the extra factor 2 takes into account that only one, not two, FP is simulated. Since $\Sigma \phi$ is the fission rate, summing over all weights, after choosing appropriate units, produces the expected total number of produced ions per $\mu \mathrm{C}$ of integrated primary beam current.

\section{Results}

Our reference simulation is based on the setup described in sect. 3 modelled by the default Geant 4 models. The main results from all simulations are summarised in table 1.

In fig. 8 we see how the mass and charge dependence of the yield of stopped ions favour light elements. Although the relative difference between the highest and lowest yield of stopped ions in the gas (gas yield) exceeds $15 \%$, the yields' mass and charge dependence between neighbouring ion species are negligible. In total, about $1.9 \%$ of the produced ions escape the target and are stopped in the gas.

Keeping the standard geometry but switching to the charge model by Schiwietz et al., gives rise to two main effects. Firstly, the stopping power increases in average and the number of ions that escape the target decreases. 
Table 1. Yields of the produced and stopped ions for the different simulations.

\begin{tabular}{llcrr}
\hline & & \multicolumn{2}{c}{ Ion yield per $\mu$ Co integrated beam current } \\
Geometry & Variation & generated & stopped in gas & stopped in walls \\
\hline Standard (fig. 4(a)) & none & $31.0 \times 10^{3}$ & $0.593 \times 10^{3}$ & $9.897 \times 10^{3}$ \\
Standard (fig. 4(a)) & Schiwietz et al.'s charge model & $31.0 \times 10^{3}$ & $0.668 \times 10^{3}$ & $7.372 \times 10^{3}$ \\
Standard (fig. 4(a)) & 400 mbar gas pressure & $31.0 \times 10^{3}$ & $1.139 \times 10^{3}$ & $9.348 \times 10^{3}$ \\
Standard (fig. 4(a)) & 600 mbar gas pressure & $31.0 \times 10^{3}$ & $1.649 \times 10^{3}$ & $8.840 \times 10^{3}$ \\
Standard (fig. 4(a)) & 800 mbar gas pressure & $31.0 \times 10^{3}$ & $2.114 \times 10^{3}$ & $8.375 \times 10^{3}$ \\
Standard (fig. 4(a)) & target thickness halved & $15.5 \times 10^{3}$ & $0.357 \times 10^{3}$ & $9.357 \times 10^{3}$ \\
Standard (fig. 4(a)) & target thickness $\times 10$ & $310.4 \times 10^{3}$ & $0.598 \times 10^{3}$ & $9.984 \times 10^{3}$ \\
Six targets (fig. 4(b)) & none & $53.0 \times 10^{3}$ & $0.994 \times 10^{3}$ & $16.918 \times 10^{3}$ \\
Single big target (fig. 4(c)) & none & $124.1 \times 10^{3}$ & $1.910 \times 10^{3}$ & $28.038 \times 10^{3}$ \\
\hline
\end{tabular}

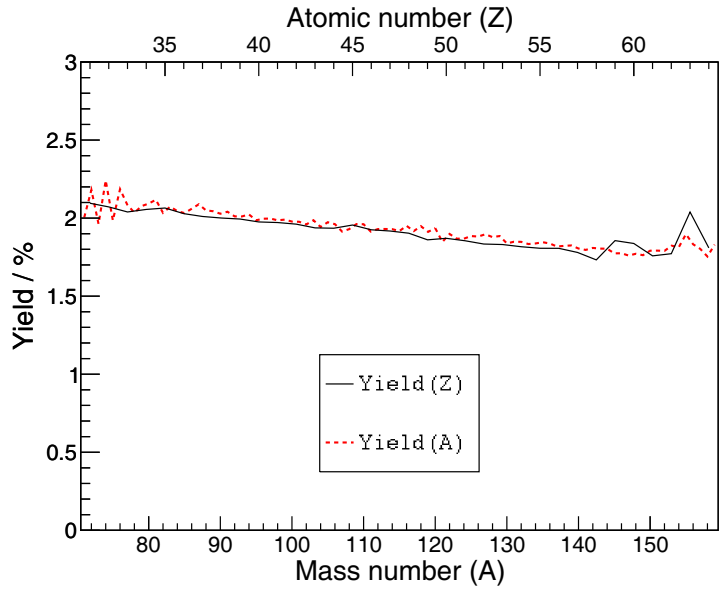

Fig. 8. Relative numbers of stopped ions from our default simulation, as a function of both mass and atomic number, respectively.

However, so does the number of ions hitting the chamber walls, and the relative number of ions stopped in the gas only increases slightly to $2.15 \%$. Secondly, the mass dependence of the gas yield increases, favouring the light masses even more than the default effective charge model (fig. 9).

Contrary to the increased mass dependence when the new effective charge model was used, the simulation with doubled gas pressure shows no change in the relative gas yields of low and high masses; it does however, increase the total gas yield almost twofold (fig. 9). In this parameter region the gas yield is close to proportional to the pressure. Presently, the pressure in the ion guide is limited to about 400 mbar by the performance of the differential pumping system, keeping the downstream beam line at high vacuum. However, increasing the pressure further in our simulations still shows no sign of an increased mass dependence (fig. 10) and gas yield increases slightly less than linearly as a function of pressure (table 1).

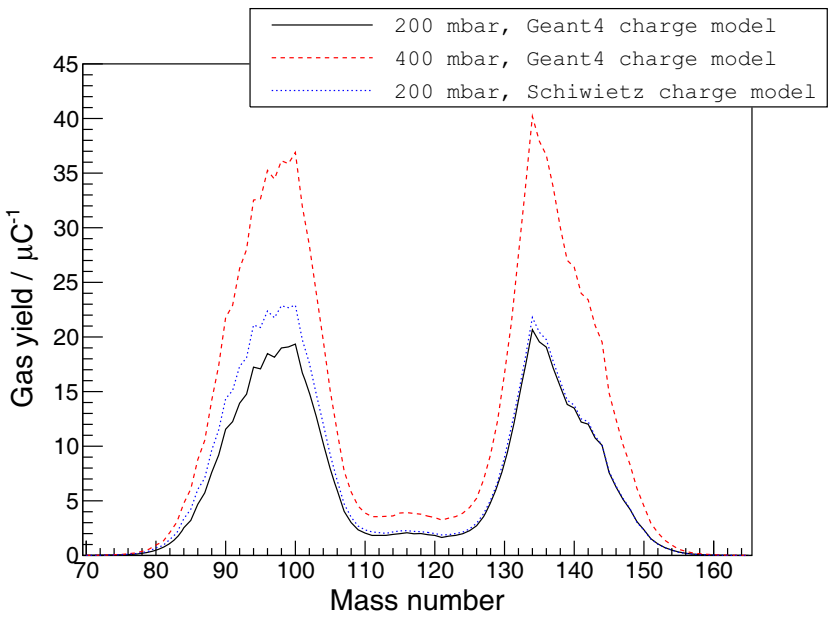

Fig. 9. Gas yield in absolute numbers for the default simulation, when the pressure was doubled to $400 \mathrm{mbar}$, and when the effective charge model was replaced by the one by Schiwietz et al. [16].

One could imagine that an increased target thickness would increase the gas yield. However, only the part of the target closest to the surface will contribute, since the FPs otherwise will not escape the target. The simulation utilising targets ten times thicker gives almost the same gas yield as the reference simulation. While an increased thickness had a vanishing effect, decreasing it by a factor of two decreases the gas yield by nearly the same factor. These two results indicate that the target thickness currently in use is close to optimal since it provides the maximum gas yield without wasting precious material. This has been extensively discussed in a previous study of the proton-induced ion guide [2].

Instead of increasing target thickness one could add more targets. The ion guide target holder was initially designed to hold up to six targets. Nonetheless, putting in four extra targets in the empty slots like in fig. 4(b), in addition to the two in fig. 4(a), does not triple the gas 


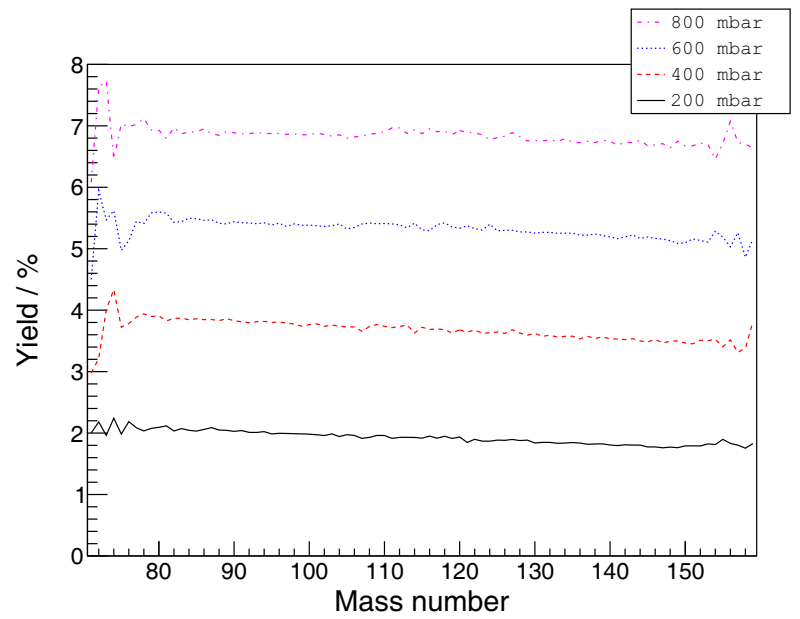

Fig. 10. Gas yield in per cent, for the default geometry, as a function of mass number. The tested buffer gas pressures were 200, 400, 600 and 800 mbar.

yield. The new targets are further away from the neutron source and the two middle ones are more off centre. In the end, each of the new targets contributes about half of what one of the targets closest to the neutron source does (see also fig. 11(b)).

A lesson learned from these results is that in order to increase the gas yield, one must increase the surface area exposed to the gas, while keeping targets, with thicknesses no smaller than the one in our reference geometry, in the high neutron flux zone. This is achieved by the big target geometry depicted in fig. 4(c). The surface area is 2.6 times larger than the combined surface area in our reference simulation, but due to its more centred position, the gas yield is 3.2 times higher (see also fig. 11(c)). The setup itself is also simpler, requiring less structures to hold the target in place.

Not only the total gas yield is important though, also where in the chamber the ions stop matters. In fig. 11 the spatial distributions of the stopped ions are depicted for the three different geometries. The spatial distribution of ions has implications on how an optimal gas flow and target distribution should look like.

To further look into new possibilities of target placement within the ion guide, we produced the cumulative distribution of ions stopped in the gas as a function of the distance to the fission event (fig. 12). The distance is measured perpendicular to the target surface, which gives us an estimate of how much free space is needed in front of each target to maximise the gas yield. Half the FPs are stopped within $14 \mathrm{~mm}$ of the target, while less than $80 \%$ are stopped within $28 \mathrm{~mm}$.

In the experiment in December 2016 [10], about nine counts per second of the isobaric chain $A=131$ were registered with a silicon detector in the switch yard after the mass-over-charge selective dipole magnet. At a proton current of $10 \mu \mathrm{A}$, and a detector efficiency of $30 \%$, this corresponds to 3 ions $/ \mu \mathrm{C}$ of primary beam. According to the simulation, 21.4 ions $/ \mu \mathrm{C}$ of this mass are stopped in the

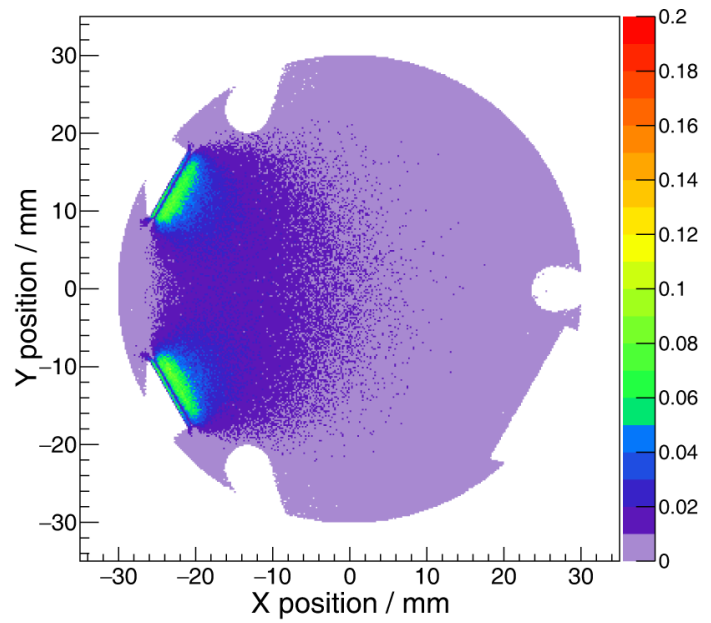

(a)

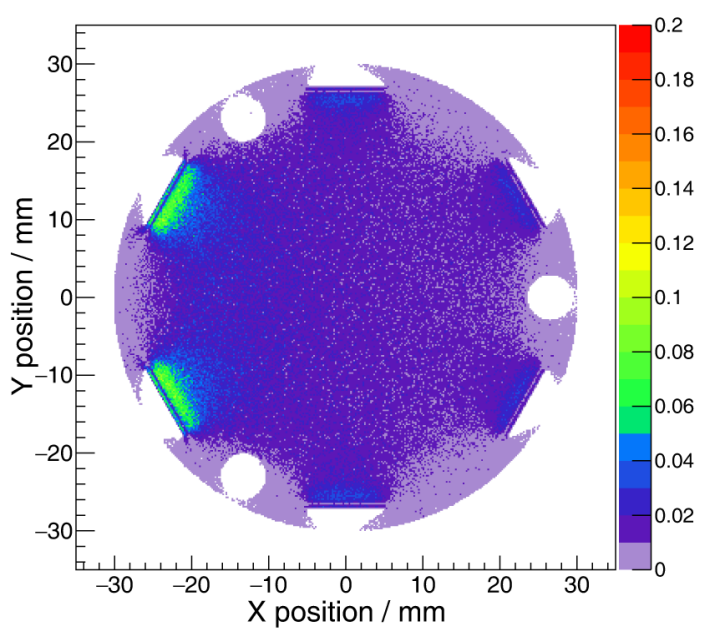

(b)

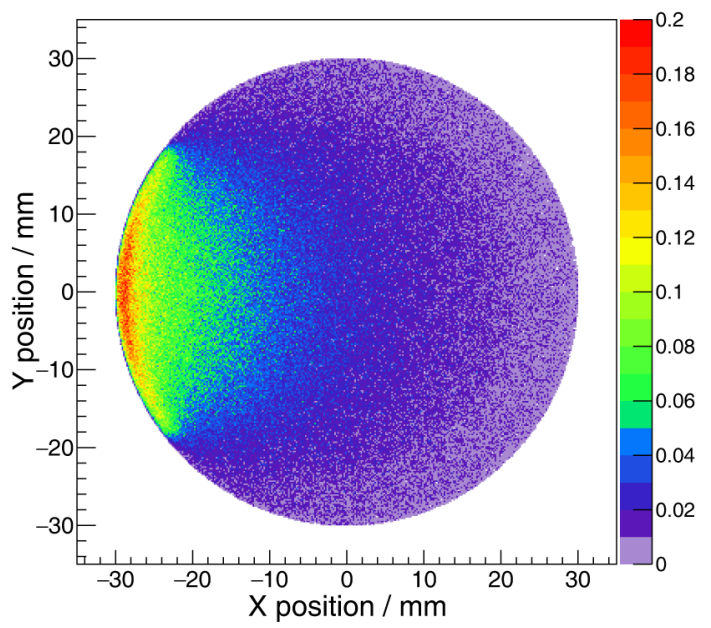

(c)

Fig. 11. Projected spatial distributions of the gas yield given per $\mu \mathrm{C}$ of integrated primary beam current. Each pixel represents a $0.2 \mathrm{~mm} \times 0.2 \mathrm{~mm}$ area. See fig. 4 for schematics of the geometries (a)-(c). 


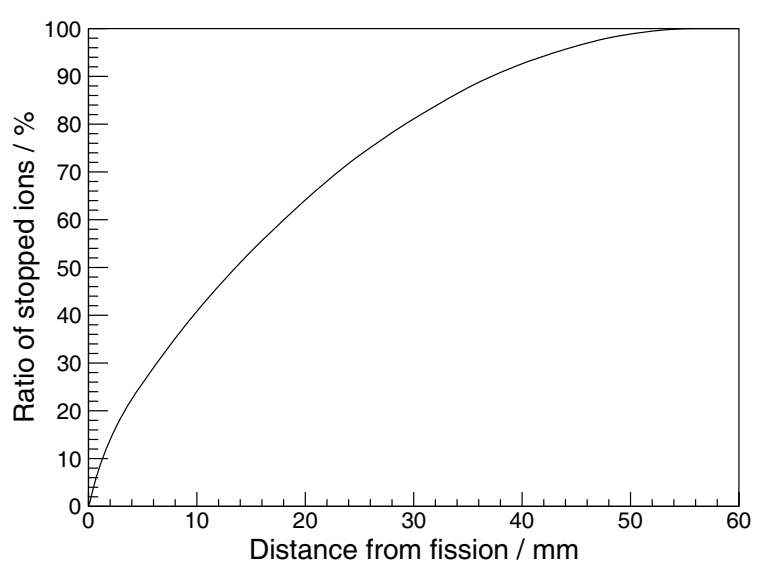

Fig. 12. Cumulative distribution of the ions stopped in the gas, as a function of distance from the fission event, measured in the plane perpendicular to the ion guide axis.

ion guide. By comparing these numbers, an ion survival probability for singly charged ions of $14 \%$ can be estimated. This efficiency includes the fraction of ions ending up as singly charged, as well as the extraction and transportation efficiency to the detector. Only the +1 ions are selected by a dipole magnet for further transport, and any ion ending up neutral or with a higher charge will be lost. A bit further downstream, at the spectroscopy station, a rate of $1.6 \mathrm{ions} / \mu \mathrm{C}$ was registered. With a detector efficiency of about $70 \%$ this corresponds to an ion survival probability of $11 \%$.

As a comparison, a record yield of 40000 ions $/ \mu \mathrm{C}$ of extracted ${ }^{112} \mathrm{Rh}$ ions was achieved in a proton-induced fission experiment in 2006 [20]. This has later been repeated, reaching 21000 ions $/ \mu \mathrm{C}$ of ${ }^{112} \mathrm{Rh}$ at the spectroscopy station [21]. According to the simulation of the ion guide for proton-induced fission, a stopping efficiency of $0.75 \%$ is expected [2]. From the independent yield of ${ }^{112} \mathrm{Rh}[8]$ and the cross section for ${ }^{238} \mathrm{U}[22]$ in proton-induced fission at $30 \mathrm{MeV}$, a partial cross section for ${ }^{112} \mathrm{Rh}$ of $15 \mathrm{mb}$ is obtained. Assuming an effective thickness of the uranium target of $t_{\mathrm{eff}}=15 \mathrm{mg} / \mathrm{cm}^{2} / \sin 7^{\circ}$ [1], the yield of ${ }^{112} \mathrm{Rh}$ in the ion guide can be estimated to 218000 ions $/ \mu \mathrm{C}$. Hence, the estimated ion survival probability becomes $18 \%$ to the switch yard and $10 \%$ to the spectroscopy station.

\section{Discussion}

Compared to the simulations of the proton-induced case [2] the methodology has improved significantly. The simulations can now guide the development of future setups in order to optimise for higher yield in the gas, making it possible to measure yields of more rare FPs. By taking realistic fission yields and the neutron flux into account, we have developed a tool to test any target configuration and get yield estimates in absolute numbers. Coming back to the tin isotopes in fig. 1 we predict the gas yield of, e.g. ${ }^{132} \mathrm{Sn}$ to be 4.3 ions $/ \mu \mathrm{C}$ in the experimental setup used in December 2016. For ${ }^{136} \mathrm{Sn}$ the corresponding gas yield is predicted to $4.7 \times 10^{-3}$ ions $/ \mu \mathrm{C}$.

Our simulations show that the single big target setup could increase the gas yield by a factor of 3.2 , compared to the current setup with nominal pressure. At the same time the pressure could be maximised to 400 mbar since no drawbacks, only higher yields, were seen doing so in neither the simulations nor the experiment in December 2016.

Even though stopping power calculations of heavy ions are associated with uncertainties of several percent (Ziegler et al. estimate an overall uncertainty of $5 \%$ [17, $18]$ ), we have shown that the gas yield remains rather constant even when the effective charge model is interchanged. We are therefore confident in the current results, and continue looking for optimised target layouts and ion guide geometries. However, the mass dependence of the gas yield was seen to increase when the effective charge model of Schiwietz et al. was used. The difference between the models was the largest for light FPs, where it reached about $10 \%$. This indicates that our results in this mass region have an increased uncertainty of the same order of magnitude. More feedback from experiments, e.g. a yield analysis of the implantation foil, could indicate which effective charge model fits reality best. In fission yield measurements, the mass dependence must be taken into account when comparing yields of non-neighbouring elements.

The estimation of the ion survival rates cannot be considered a benchmark, since two simulations are compared against each other. To truly benchmark the simulations a direct comparison against measured data, for example from the implantation foil in the ion guide, will be necessary.

The difference in ion survival probability between the proton and neutron-induced cases could be explained by the different geometries of the two ion guides. Also important for the ion survival is the chemical dependence. In the proton-induced case rhodium is studied, while in the neutron-induced case the isobar $A=131$ includes several elements, predominantly antimony, tin and tellurium. An element dependence of the charge exchange probability with the helium gas could hence play a significant role.

We thank the IGISOL groups both in Uppsala and Jyväskylä for their hard experimental work and fruitful discussions. Funding for this work was received from the Swedish Nuclear Radiation Safety Authority and the Swedish Nuclear Fuel and Waste Management Co.

Open Access This is an open access article distributed under the terms of the Creative Commons Attribution License (http://creativecommons.org/licenses/by/4.0), which permits unrestricted use, distribution, and reproduction in any medium, provided the original work is properly cited. 


\section{References}

1. Juha Äystö, Tommi Eronen, Ari Jokinen, Anu Kankainen, Iain Moore, Heikki Penttilä (Editors), Hyperfine Interactions, Three decades of research using IGISOL technique at the University of Jyväskylä, Vol. 223 (Jan. 2014).

2. A. Al-Adili et al., Eur. Phys. J. A 51, 59 (2015).

3. A. Mattera et al., Eur. Phys. J. A 53, 173 (2017).

4. A. Solders et al., Nucl. Data Sheets 119, 338 (2014).

5. J. Van Schelt et al., Phys. Rev. Lett. 111, 061102 (2013).

6. G. Lhersonneau et al., Eur. Phys. J. A 9, 385 (2000).

7. G.W. McKinney et al., MCNPX 2.5.0 - New Features Demonstrated, in Proceedings of the MC2005 Conference, Chattanooga, Tennessee, April 17-21, 2005 (American Nuclear Society, 2005) LA-UR-04-8695.

8. K.-H. Schmidt et al., Nucl. Data Sheets 131, 107 (2016).

9. S. Agostinelli et al., Nucl. Instrum. Methods A 506, 250 (2003).

10. A. Mattera et al., Production of Sn and Sb isotopes in highenergy neutron induced fission of ${ }^{\text {nat }} U$, in preparation.

11. M.B. Chadwick et al., Nucl. Data Sheets 112, 2887 (2011).

12. P.G. Young et al., Transport data libraries for incident proton and neutron energies to $100 \mathrm{MeV}$, Technical Report LA-11753-MS, Los Alamos National Laboratory (1990).
13. P.F. Rose, ENDF/b-vi summary documentation. Technical Report BNL-NCS-17541, 4th Edition, Brookhaven National Laboratory October 1991.

14. S.C. Frankle, Summary documentation for the endlg2 continuous-energy neutron data library (release 1), Technical Report XTM:96-05 and LA-UR-96-327, Los Alamos National Laboratory (1996).

15. R. Brun, F. Rademakers, Nucl. Instrum. Methods A 389, 81 (1996).

16. G. Schiwietz, P.L. Grande, Nucl. Instrum. Methods B 175, 125 (2001)

17. J.F. Ziegler, J.M. Manoyan, Nucl. Instrum. Methods B 35, 215 (1988)

18. J.F. Ziegler, M.D. Ziegler, J.P. Biersack, Nucl. Instrum. Methods B 268, 1818 (2010).

19. P. Constantin, D.L. Balabanski, P.V. Cuong, Nucl. Instrum. Methods Phys. Res. B 372, 78 (2016).

20. Department of Physics, Jyväskylä University, JYFL Annual Report 2006, available online: https://www.jyu.fi/ static/fysiikka/vuosikertomukset/annual2006.pdf.

21. H. Penttilä, Fission yield measurements at the renoved IGISOL-4 facility, JEF/DOC-1580, JEFF (2014).

22. K. Shibata et al., J. Nucl. Sci. Technol. 48, 1 (2011). 